\title{
Transitional properties of droughts and related impacts of climate indices in the Pearl River basin, China
}

Mingzhong Xiao, Qiang Zhang, Vijay P. Singh, Lin Liu

\section{Corresponding author:}

Qiang Zhang, Ph.D. Professor

Department of Water Resources and Environment (Department Chairman)

SunYat-sen University

Guangzhou 510275,

China

Tel: +86-20-84113730

Fax: +86-20-84113730

E-mail: zhangq68@ mail.sysu.edu.cn (preferred contact address) 


\title{
Transitional properties of droughts and related impacts of climate indices in the Pearl River basin, China
}

\author{
Mingzhong Xiao ${ }^{1,2}$, Qiang Zhang ${ }^{1,2,3}$, Vijay P. Singh ${ }^{4}$, Lin Liu ${ }^{3,5}$
}

1. Department of Water Resources and Environment, Sun Yat-sen University, Guangzhou 510275, China;

2. Key Laboratory of Water Cycle and Water Security in Southern China of Guangdong High Education Institute, Sun Yat-sen University, Guangzhou 510275, China;

3. Guangdong Provincial Key Laboratory of Urbanization and Geo-simulation, School of Geography and Planning, Sun Yat-sen University, Guangzhou 510275, China;

4. Department of Biological \& Agricultural Engineering and Zachry Department of Civil Engineering, Texas A \& M University, College Station, Texas 77843-2117, USA.

5. Department of Geography, University of Cincinnati, Cincinnati, OH 45221-0131, USA.

\begin{abstract}
Drought is the natural hazard poorly understood so far due to various mechanisms behind. Moreover, disastrous effects of drought on human society necessitate accurate forecasting of drought behaviors. In this case, to improve forecasting of drought in the Pearl River basin, a trivariate copula model has been developed and used to include the El Nino Southern Oscillation (ENSO), North
\end{abstract}


Atlantic Oscillation (NAO), Indian Ocean Dipole (IOD) and Pacific Decadal Oscillation (PDO) into model structure of Markov chain. The standardized precipitation evapotranspiration index (SPEI) has been used to monitor the drought in this study. Comparison with the preliminary correlation analysis between each month climate index and SPEI series indicated that the trivariate copula performs satisfactorily well in evaluation of influences of climate indices on the transition probabilities of drought. It is considered that the region with the negative vertical velocity is dominated by more precipitation and vice versa. Moreover, field patterns of $500 \mathrm{hPa}$ vertical velocity anomalies related to each climate index have further corroborated the influences of climate indices on the drought behaviors. Besides, the mean extreme drought durations under different conditions of each climate index have also been investigated in this study. Results indicated that the mean extreme drought duration tends to be longer in the western part of the Pearl River basin during positive phase of ENSO while tends to be shorter during the positive phase of NAO and vice versa; in the central part of the Pearl River basin, the mean extreme drought duration tends to be shorter during the positive phase of ENSO, NAO and PDO while tends to be longer during the positive episode of IOD, and vice versa; in the eastern part of the Pearl River basin, the mean extreme drought duration tends to be shorter during the positive episode of ENSO and PDO, and vice versa. This study sheds new light on transitional behaviors of droughts as a result of influences from climate indices. 
Key words: Drought behaviors; Markov chain; Trivariate copula; Standardized precipitation evapotranspiration index (SPEI); Climate indices

\section{Introduction}

In recent decades, given changing climatic and documented increases in extreme climate events, human concerns has grown worldwide pertaining to the fact that droughts have the potential to intensify in frequency, severity and duration (Peterson et al., 2013; Wilhite et al., 2014). As a prolonged water deficit event, drought has a devastating effect on agriculture, energy production, urban water supply, public health, ecosystem, and the economy. It is estimated that the USA economic losses caused by droughts are as high as 6 to 8 billion dollars each year, being far more than other meteorological disasters (Wilhite, 2000). However, unlike other extreme climate events such as floods, earthquakes, hurricanes, the evolution of drought is slow, then an effective mitigation of the most adverse drought impacts is possible by capitalizing on the delay between the onset of drought and the moment when its consequences are perceived by the water supply systems or the end-users (Bonaccorso et al., 2015).

Accurate forecasting of drought plays a significant role in developing appropriate policies to mitigate the drought hazards, and the ability to accurately forecast the onset, persistence and cessation of drought conditions is fundamental to the development and implementation of efficient mitigation strategies. In recent years, many statistical and non-statistical techniques have been proposed for the forecasting 
of drought (Bonaccorso et al., 2015; Madadgar and Moradkhani, 2013; X Yuan et al., 2013). Based on the Climate Forecast System, version 2 (CFSv2) and the Variable Infiltration Capacity (VIC) land surface model, Yuan et al. (2013) have established a seasonal hydrologic forecasting system for Africa. The 2012 drought was one of the most extensive drought events in the United States, resulting in the economic loss over 12 billion USD, AghaKouchak (2014) found that this drought was predictable several months in advance based on the persistence property of accumulated soil moisture. In addition, with the copula function to model the autocorrelated streamflow or hydrologic drought, a multivariate probabilistic approach to perform drought forecasting within a Bayesian framework has been proposed by Madadgar and Moradkhani (2013) that a future drought status can be estimated given the earlier drought conditions.

Regardless of the specific methodology, Bonaccorso et al. (2015) stated that a distinction can be made with reference to the different objectives of the drought forecasting. This current study focuses on determining transition probabilities from a given current drought class to another one in the future, and this has been largely evaluated by the Markov chain models (Bonaccorso et al., 2015; Cancelliere et al., 2007; Madadgar and Moradkhani, 2013; Paulo and Pereira, 2007; Sanusi et al., 2015). In our previous studies (Chen et al., 2015), with the drought monitored by the Standardized Precipitation Evapotranspiration Index (SPEI) with the time scale of 3 months, the drought characteristics of the Pearl River basin has been analyzed using the Markov chain. Due to the limited sample size of observed transitions among 
different drought classes, it has been found that the lack of observed transitions in some cases would lead to the misleading conclusion that such transitions have zero occurrence probability when the transition probability matrix was estimated by the maximum likelihood method (Cancelliere et al., 2007; Chen et al., 2015). To avoid these drawbacks of the maximum likelihood method, a method to theoretically calculate the transition probability matrix based on the bivariate copula function has been introduced in our previous paper (Chen et al., 2015).

Despite such efforts, the forecasting of drought is still a tough task. Recently, much attention has been attached to forecasting of drought behaviors using climate indices as main predictors, such as the El Niño Southern Oscillation (ENSO) (S T Chen et al., 2013) and North Atlantic Oscillation (NAO) (Bonaccorso et al., 2015). With advances in the field of synoptic climatology, it has been found that ocean-atmosphere interactions are not chaotic or random, and influences of global climatic indices on weather and hydrological extremes have been drawing increasing concerns (Cai et al., 2011; Dai, 2013; Moore et al., 2013; Xiao et al., 2015; Zhang et al., 2014). Located in the south China, the climate of the Pearl River basin is dominated by the East Asian Monsoon, it has also been well studied that the East Asian Monsoon has been significantly influenced by the ENSO, NAO, Indian Ocean Dipole (IOD) and Pacific Decadal Oscillation (PDO) (W Chen et al., 2013; Linderholm et al., 2011; Xiao et al., 2015; Y Yuan et al., 2008; Zhang et al., 2014). In our previous studies, the possible influences of ENSO, NAO, IOD and PDO on the seasonal precipitation in the Yangtze River basin (Xiao et al., 2015) and the 
Poyang Lake basin (Zhang et al., 2014) have been analyzed. In Sicily (Italy), with NAO as exogenous input variable, Bonaccorso et al. (2015) found that the probabilistic models of Markov chain perform better for short and middle term forecasting of drought transition probabilities. Then it is also necessary to encompass the ENSO, NAO, IOD and PDO into the models structure of Markov chain to improve the drought forecasting in the Pearl River basin. However, such reports have not been available so far which to some degree limit human mitigation to droughts in the Pearl River basin.

As an extension of our previous study (Chen et al., 2015), a trivariate copula has been developed and used to model the dependence between two consecutive monthly SPEI and each climate index, and then the transition probability matrix under different conditions of each climate index can be calculated, details of the calculation can be referred to Section 3. With respect to the case when the climate index is neglected, the enhancement in forecasting drought transition probabilities has been quantified in the study. Furthermore, based on the NCAR/NCEP reanalysis dataset, the $850 \mathrm{hPa}$ wind anomalies and $500 \mathrm{hPa}$ vertical velocity anomalies related to each climate index have been investigated to verify the results, and these will be important to further understand the possible geophysical processes linking the teleconnections of each climate index to monthly drought in the Pearl River basin. In addition, the paper is organized as follows: introduction of study region and data is presented in Section 2; methods of analysis are depicted in Section 3; and results and discussion thereof are described in Section 4, which is followed by Section 5 as the 
conclusion of this study.

\section{Study region and data}

Located in the south China, the Pearl River $\left(102^{\circ} 14^{\prime} \mathrm{E}-115^{\circ} 53^{\prime} \mathrm{E}\right.$; $\left.21^{\circ} 31^{\prime} \mathrm{N}-26^{\circ} 49^{\prime} \mathrm{N}\right)$ is the second largest river in terms of the water volume and the third largest river in terms of the drainage basin area in China. The Pearl River basin is $4.573 \times 10^{5} \mathrm{~km}^{2}$ in drainage area with three major tributaries: the West River, the North River and the East River (Fig. 1). As located in the tropical and sub-tropical climate zones, the annual mean temperature of the Pearl River basin ranges from $14^{\circ} \mathrm{C}$ to $22^{\circ} \mathrm{C}$, with long-term annual average precipitation of $1525.1 \mathrm{~mm}$. The distribution of precipitation is uneven during the year, and precipitation from April to September accounts for $80 \%$ of the annual total (Chen et al., 2015; Zhang et al., 2012).

Obtained from the National Meteorological Information Center of the China Meteorological Administration, daily precipitation data and $2 \mathrm{~m}$ air temperature (maximum temperature and minimum temperature) data were analyzed, covering a period of 1959-2013 at 46 meteorological stations across the Pearl River basin. Detailed information of the meteorological data analyzed in this study can be referred to Chen et al. (2015). Locations of these meteorological stations are shown in Fig. 1. In addition, as the climate across the Pearl River basin is complicated and diverse, the Pearl River basin has been subdivided into three sub-regions, i.e. the western, central and eastern part of the Pearl River basin (Fig. 1). Same as the data used in our previous study (Xiao et al., 2015), the ENSO are indicated by Niño 3.4 indices in the 
study, derived from sea surface temperature anomaly estimates in the Niño 3.4 region $\left(5^{\circ} \mathrm{N}-5^{\circ} \mathrm{S}, 120^{\circ} \mathrm{W}-170^{\circ} \mathrm{W}\right)$, and details of the sources for the ENSO, NAO, IOD and PDO can be referred to Xiao et al. (2015).

\section{Methodology}

\subsection{Standardized Precipitation Evapotranspiration Index (SPEI)}

As an extension of the widely used Standardized Precipitation Index (SPI), the SPEI has been proposed by Vicente-Serrano et al. (2010) to analyze the drought, which can capture the effect of increased temperatures on water demand. The SPEI is designed to take into account both precipitation and potential evapotranspiration (PET) in determining drought. PET is the amount of evaporation and transpiration that would occur if a sufficient water were available. The SPEI with the time scale of 3 months has been calculated in the Pearl River basin to analyze the drought (Chen et al., 2015), and the 3-month SPEI was also accepted in this study for drought monitoring. Besides, the SPEI-based drought classification (Chen et al., 2015) has also been used in the study (Table 1), and the drought classification is a modification of the drought classification determined by the U.S. Drought Monitor.

\subsection{Trivariate copula functions}

Copula functions have been widely used (Genest et al., 2011; Kao and Govindaraju, 2010; Madadgar and Moradkhani, 2013; 2014; Nelsen, 2006; Zhang et al., 2012; Zhang et al., 2013) due to the fact that Copula functions can model the 
dependence structure between random variables irrespective of the types of marginal involved. In this study, six copula functions, i.e. the Archimedean (Gumbel, Clayton and Frank), Elliptical (Gaussian and t) and Plackett copulas (referred to Table 2 of Chen et al., 2015) have been used to appropriately join the marginal distributions of two adjacent monthly drought in the Pearl River basin. However, the monthly drought may also be influenced by the climate indices, then it is necessary to model the dependence of the trivariate dependent variables with the trivariate copula.

To derive a multivariate frequency analysis, the Gaussian copula, belong to the family of Metaelliptical copulas, has been introduced by Renard and Lang (2007). It is a convenient model for multidimensional $(>2)$ variables, however, it also suffers from other limitations, details referred to Renard and Lang (2007) and Kao and Govindaraju (2008), so in the paper, the Metaelliptical copulas have not been used for the trivariate copula. Based on the conditional approach suggested by Chakak and Koehler (1995) to construct multivariate models, Salvadori and De Michele (2006) performed a trivariate storm frequency analysis in which all pairwise dependencies could be preserved. The structure of the trivariate copula is defined that (Salvadori and De Michele, 2006):

$$
C_{I W D}(r, s, t)=t C_{I W}\left(\frac{C_{I D}(r, t)}{t}, \frac{C_{W D}(s, t)}{t}\right)
$$

where $\mathrm{r}, \mathrm{s}, \mathrm{t} \in(0,1)$. The trivariate function $\mathrm{C}_{\mathrm{IWD}}$ has three two-dimensional marginals given by the bivariate copulas $\mathrm{C}_{\mathrm{IW}}, \mathrm{C}_{\mathrm{ID}}$ and $\mathrm{C}_{\mathrm{WD}}$. The two arguments of $\mathrm{C}_{\mathrm{IW}}$ in Eq. (1) are conditional distributions, thus provide the link between I and $\mathrm{W}$, given the behavior of $\mathrm{D}$. Besides, there are also some drawbacks for this trivariate 
copula, and it has been argued by Kao and Govindaraju (2008) that the trivariate copula cannot provide a unique distribution if the sequence of variables is altered. However, we only focus on the dependence structure between two adjacent monthly drought given the conditions of climate indices in the study. In this case, this drawback will not limit the use of the trivariate copula. In the study, the variable D is used to represent the climate index while the variables I and $\mathrm{W}$ are used to represent the two adjacent monthly drought. Furthermore, compared to the trivariate plackett copula proposed by Kao and Govindaraju (2008), the structure of the trivariate copula defined in Eq. (1) is particularly simple and appealing. In addition, the parameters of $\mathrm{C}_{\mathrm{IWD}}$ are only those of its marginals, and no further estimations are required to fit $\mathrm{C}_{\mathrm{IWD}}$ (Salvadori, 2007; Salvadori and De Michele, 2006).

\subsection{The transition probability derived by the copula function}

Due to the limited sample size of observed transitions among different drought classes, the validity of a Markov chain model with the transition probability matrix formed based on the maximum likelihood method has been questioned by Cancelliere et al. (2007) that the lack of observed transitions in some cases would lead to the misleading conclusion that such transitions have zero occurrence probability. To avoid these drawbacks of the maximum likelihood method, a method to theoretically calculate transition probability matrix based on the bivariate copula functions has been introduced in our previous paper (Chen et al., 2015). It can be expressed as a conditional probability that (Cancelliere et al., 2007; Chen et al., 
2015):

$$
P\left(X \in X_{j} \mid Y \in Y_{i}\right)=\frac{P\left(X \in X_{j}, Y \in Y_{i}\right)}{P\left(Y \in Y_{i}\right)}
$$

However, the monthly drought may also be influenced by the climate indices, and then the conditional probability defined in Eq. (2) will change under the different conditions of climate index. This can be expressed as a double conditional probability that $\mathrm{P}\left(\mathrm{X} \in \mathrm{X}_{\mathrm{j}} \mid \mathrm{Y} \in \mathrm{Y}_{\mathrm{i}}, \mathrm{Z} \in \mathrm{Z}_{\mathrm{k}}\right)$, and based on the Eq. (2), it can be calculated as follow:

$$
\begin{aligned}
P\left(X \in X_{j} \mid Y \in Y_{i}, Z \in Z_{k}\right)=\frac{P\left(X \in X_{j}, Y \in Y_{i} \mid Z \in Z_{k}\right)}{P\left(Y \in Y_{i} \mid Z \in Z_{k}\right)} & P\left(X \in X_{j}, Y \in Y_{i}, Z \in Z_{k}\right) / P\left(Z \in Z_{k}\right) \\
= & \frac{P\left(X \in X_{j}, Y \in Y_{i}, Z \in Z_{k}\right)}{P\left(Y \in Y_{i}, Z \in Z_{k}\right)}
\end{aligned}
$$

Assuming that the lower and upper bounds of $\mathrm{X}_{\mathrm{j}}, \mathrm{Y}_{\mathrm{i}}$ and $\mathrm{Z}_{\mathrm{k}}$ are denoted by $\mathrm{x}_{1}, \mathrm{x}_{2}$, $\mathrm{y}_{1}, \mathrm{y}_{2}, \mathrm{z}_{1}$ and $\mathrm{z}_{2}$ respectively, and $\mathrm{u}_{1}=\mathrm{F}_{\mathrm{X}}\left(\mathrm{x}_{1}\right), \mathrm{u}_{2}=\mathrm{F}_{\mathrm{X}}\left(\mathrm{x}_{2}\right), \mathrm{v}_{1}=\mathrm{F}_{\mathrm{Y}}\left(\mathrm{y}_{1}\right), \mathrm{v}_{2}=\mathrm{F}_{\mathrm{Y}}\left(\mathrm{y}_{2}\right)$, $\mathrm{z}_{1}=\mathrm{F}_{\mathrm{Z}}\left(\mathrm{z}_{1}\right), \mathrm{z}_{2}=\mathrm{F}_{\mathrm{Z}}\left(\mathrm{z}_{2}\right)$, then the Eq. (3) can be further expressed as that:

$$
\begin{gathered}
P\left(X \in X_{j} \mid Y \in Y_{i}, Z \in Z_{k}\right)=\frac{A}{C_{Y Z}\left(v_{2}, z_{2}\right)-C_{Y Z}\left(v_{2}, z_{1}\right)-C_{Y Z}\left(v_{1}, z_{2}\right)+C_{Y Z}\left(v_{1}, z_{1}\right)} \\
\text { where } \begin{array}{c}
A= \\
\quad C_{X Y Z}\left(u_{2}, v_{2}, z_{2}\right)-C_{X Y Z}\left(u_{2}, v_{1}, z_{2}\right)-C_{X Y Z}\left(u_{1}, v_{2}, z_{2}\right)+C_{X Y Z}\left(u_{1}, v_{1}, z_{2}\right) \\
\\
-C_{X Y Z}\left(u_{2}, v_{2}, z_{1}\right)+C\left(u_{2}, v_{1}, z_{1}\right)+C\left(u_{1}, v_{2}, z_{1}\right)-C\left(u_{1}, v_{1}, z_{1}\right)
\end{array}
\end{gathered}
$$

It should be noted here that the denominator of Eq. (4) can be considered as a calculation of the area for the rectangle defined by the bounds of $Y_{i}$ and $Z_{k}$, that $\left(\mathrm{v}_{2}-\mathrm{v}_{1}\right) *\left(\mathrm{z}_{2}-\mathrm{z}_{1}\right)=\mathrm{v}_{2} * \mathrm{z}_{2}-\mathrm{v}_{2} * \mathrm{z}_{1}-\mathrm{v}_{1} * \mathrm{z}_{2}+\mathrm{v}_{1} * \mathrm{z}_{1}$; and the numerator of Eq. (4) can be considered as a calculation of the volume for the cube defined by the bounds of $\mathrm{X}_{\mathrm{j}}$, $\mathrm{Y}_{\mathrm{i}}$ and $\mathrm{Z}_{\mathrm{k}}$, that $\left(\mathrm{u}_{2}-\mathrm{u}_{1}\right) *\left(\mathrm{v}_{2}-\mathrm{v}_{1}\right) *\left(\mathrm{z}_{2}-\mathrm{Z}_{1}\right)=\mathrm{u}_{2} * \mathrm{v}_{2} * \mathrm{z}_{2}-\mathrm{u}_{2} * \mathrm{v}_{1} * \mathrm{Z}_{2}-\mathrm{u}_{1} * \mathrm{v}_{2} * \mathrm{z}_{2}+\mathrm{u}_{1} * \mathrm{v}_{1} * \mathrm{Z}_{2}$ 
$-\mathrm{u}_{2} * \mathrm{v}_{2} * \mathrm{z}_{1}+\mathrm{u}_{2} * \mathrm{v}_{1} * \mathrm{z}_{1}+\mathrm{u}_{1} * \mathrm{v}_{2} * \mathrm{z}_{1}-\mathrm{u}_{1} * \mathrm{v}_{1} * \mathrm{z}_{1}$

\section{Results and discussion}

4.1 The relationship between each monthly climate index and SPEI

To analyze the relationship between each monthly climate index and SPEI, the Pearson correlations between each monthly climate index and the SPEI in the next month have been calculated. As the climate across the Pearl River basin is complicated and diverse, the Pearl River basin has been divided into three sub-regions that the Western, Central and Eastern part, and the Pearson correlations between each monthly climate index and the SPEI in the next month are different in each sub-region (Fig. 2). It can be seen from Fig. 2 that not all of the monthly SPEI have been significantly influenced by the climate indices, the SPEI in the spring (March, April and May) and autumn-winter (November, December and January) are more significantly influenced by the climate indices, and there are also much larger difference between the three sub-regions in the spring and autumn-winter. During October, November and December, both the ENSO and IOD have larger influence on the SPEI, and the positive influences are generally much stronger in the Central part. In addition, the climate of the Pearl River basin is dominated by the East Asian Monsoon which generally starts at April, and it has been found that the East Asian Monsoon has been significantly influenced by the ENSO, IOD, NAO and PDO, so it can be expected that the SPEI in the spring time is also greatly influenced by the ENSO, IOD, NAO and PDO (shown in Fig. 2), however, the influences are much 
difference in each sub-region. The influences of ENSO and PDO on the SPEI in spring are the same, with the relationship generally changes from the negative to the positive for the sub-regions from the Western part to the Eastern part. On the contrast, the relationship between IOD, NAO and the SPEI in spring generally decreased for the sub-regions from the Western part to the Eastern part, however, the IOD generally has the negative impact on the SPEI while the NAO has the positive impact on the SPEI.

4.2 The variation of transition probabilities under different conditions of each climate index

To evaluate the influences of climate indices on the transition probabilities of Markov Chain, a trivariate copula has been used in the study, and the transition probabilities matrix under different conditions of each climate index have been calculated. It should be noted here that the applicability of the copula functions have been evaluated by the same goodness of fit test method used by (Chen et al., 2015). It has been found that the Clayton, Gumbel and t copulas perform well in modeling the dependences structures between the monthly climate indices and SPEI-3, while the Gumbel, Gaussian and t copulas perform well in modeling the dependences structures between two consecutive monthly SPEI-3, the results have not been shown in the paper. With the appropriate copulas have been selected to model the pair-wise dependencies for the three variables, the trivariate copula can be constructed as introduced in section 3.2. It should be noted here that for the stations 
in some months without the appropriate copula functions to model the pair-wise dependencies, it is impossible to construct the trivariate copula, and then the transition probabilities are set as null. It has been found in Fig. 2 that the climate indices in March have larger influences on the SPEI in the April, then the March has been considered as a typical month for the evaluation of the influences of climate indices on the transition probabilities of Markov Chain, and the transition probabilities from No drought status to No drought status (the definition of drought status referred to Table 1) in March have been illustrated in Fig. 3. It can be seen from Fig. 3 that the transition probabilities from No drought status to No drought status generally tends to increase with the increasing of ENSO, NAO and PDO, while decrease with the increasing of IOD, and these are consistent with the results in Fig. 2. Specifically, for the sub-regions from the Western part to the Eastern part, it can also be seen from Fig. 3A that the positive relationships between the transition probabilities from No drought status to No drought status and the ENSO are more significant in the Eastern part, and these variation of transition probabilities from No drought status to No drought status in different sub-regions are also in line with the influences of the IOD, NAO and PDO (Fig. 2). So it can be concluded that the trivariate copula is useful for the evaluation of the influences of climate indices on the transition probabilities of Markov Chain, and this is also necessary when the possible geophysical processes linking the influence of climate indices on the month precipitation has been illustrated in the paper.

To clarify the influences of climate indices in March on the precipitation in April, 
based on the NCAR/NCEP reanalysis dataset, the field patterns of $850 \mathrm{hPa}$ wind anomalies and $500 \mathrm{hPa}$ vertical velocity anomalies with respect to each climate index have been illustrated in Fig. 4. A negative vertical velocity means ascending motion, that is there are worm advections, and these will provide the conditions for the precipitation, so it is considered that the region with the negative vertical velocity is dominated by more precipitation and vice versa. It can be seen from Fig. 4 that the Pearl River basin is dominated by the negative vertical velocity in April corresponding to the positive phase of ENSO, NAO and PDO in March, and these further prove that the positive influences of ENSO, NAO and PDO in March on the precipitation in April. The negative influences of IOD in March on the precipitation in April should be owing to the cold advection (positive vertical velocity) in that month corresponding to the positive phase of IOD in March (shown in Fig. 4B). Besides, it has been illustrated in the foregoing that the influences of climate indices on the SPEI are different in each sub-region, and these can also be reflected in Fig. 4. The vertical velocity is varied in different regions of the Pearl River basin. It can be seen from Fig. 4B that the positive vertical velocity is increased from the Western part to the Eastern part corresponding to the positive phase of IOD in March, which will cause less precipitation in the Eastern part, then this further verify that the IOD has much larger negative influence on the SPEI in April at the Eastern part. For the NAO and PDO, the possible cause of the variation of their influences on the SPEI in different regions of the Pearl River basin can also be clearly found in Fig. 4. Corresponding to the positive phase of NAO in March, the warm advection 
(negative vertical velocity) is weakened from the Western part to the Eastern part, and then there will be less precipitation in the Western part in April. However, the warm advection is enhanced from the Western part to the Eastern part corresponding to the positive phase of PDO in March, and this caused that more precipitation in the Eastern part of the Pearl River basin in April.

\subsection{The forecasted probabilities of drought under different conditions of each climate index}

After the transition probabilities matrix of Markov chain have been calculated, we can forecast the probabilities of drought in the future given the drought status in the earlier time. In this study, the initial probability of each drought status is assumed the same in the earlier time, that is $1 / 6$ as there are six drought status (referred to Table 1). For the Markov chain, the probabilities of drought status in one month ahead can be forecasted as that $\mathrm{p}_{1}=\mathrm{p}_{0} \mathrm{P}$, where the $\mathrm{P}$ is the transition probabilities matrix and $\mathrm{p}_{0}$ is the initial probability of each drought status in the previous month. In the study, the occurrence probabilities of drought in April under different conditions of each climate index have been calculated, and for each sub-region, the regional average probabilities of each drought status have been shown in Fig. 5. As it has been found that the transition probabilities from No drought status to No drought status generally increased with the increasing of ENSO, NAO and PDO while decreased with the increasing of IOD in March, it can be expected that the forecasted occurrence probabilities of No drought status increased with the increasing of ENSO, 
NAO and PDO while decreased with the increasing of IOD (shown in Fig. 5), and the occurrence probabilities of D4 is decreased with the increased of the occurrence probabilities of No drought while no large difference for the occurrence probabilities of D0, D1, D2 and D3. Besides, due to the variation of the transition probabilities matrix in different sub-regions when corresponding to different climate indices, the forecasted occurrence probabilities of each drought status are also varied in each sub-region under different conditions of climate indices, especially for the No drought status and D4.

For each month, the forecasted regional average occurrence probabilities of No drought status under different conditions of each climate index have also been calculated as shown in Fig. 6. Compared to Fig. 2, it can be seen from Fig. 6 that the variation of the forecasted occurrence probabilities of No drought status are consistent with the variation of the relationship between each month climate index and SPEI in the next month, and there are larger difference for the occurrence probabilities of No drought under different conditions of climate indices when the relationships are more significant. These further indicate that the dependence between each month climate index and SPEI have been appropriately modeled in the trivariate copula.

4.4 The calculated mean drought duration under different climate conditions

As shown in Table 1, six drought status have been classified, and in the study a drought event with the severity not less than a certain drought status has been 
considered as a certain severe drought. With this definition, three drought characteristics have been defined in our previous paper (referred to Fig. 1 of Chen et al., 2015) that the mean drought duration, the mean first passage time from a certain drought status to no drought and the mean first time from no drought to a certain severe drought. To evaluate the influence of climate index on the drought characteristic, the mean durations of extreme drought (the drought with the severity not less than D3) under different conditions of each climate index have been calculated in the study. It has been noted in section 4.2 that for the stations in some months without the appropriate copula functions to model the pair-wise dependencies, the transition probabilities are set as null. However, to calculate the duration for a station, all of the months should have the transition probabilities matrix, then the average of transition probabilities matrix in other months has been assigned for the month with null transition probability in the study.

It can be seen from Fig. 7 that the regional responses of the drought on the ENSO are different, and the mean extreme drought duration varied under different conditions of ENSO. Generally, the mean extreme drought duration tends to increase with the increasing of ENSO in the Western part while decrease with the increasing of ENSO in the Central and Eastern part of the Pearl River basin. Visual comparison of the spatial patterns of the mean extreme drought duration in the Pearl River basin under different conditions of IOD, it can be seen from Fig. 8 that the mean extreme drought duration in the Central part of the Pearl River basin has been significantly influenced by the IOD, and the mean extreme drought duration tends to increase 
with the increasing of the IOD. Opposite to the results found in the Sicily region (Italy) that the increasing of NAO tends to increase the occurrence probability of drought (Bonaccorso et al., 2015), it has been found in Fig. 9 that the mean extreme drought duration in the Western and Central part of the Pearl River basin has been significantly influenced by the NAO, and the mean extreme drought duration tends to decrease with the increasing of the NAO. The PDO is most frequently referred to a long-lived El-Niño like pattern of the Pacific climate variability. Compared to Figs. 7 and 10, it can be found that the influence of PDO on the mean extreme drought duration is similar to the results of ENSO. The mean extreme drought duration in the Central and Eastern part of the Pearl River basin tend to decrease with the increasing of the PDO, however, the influence of PDO on the mean extreme drought duration in the Western part is not significant.

\section{Conclusion}

To encompass the ENSO, NAO, IOD and PDO into the models structure of Markov chain to improve the forecasting of drought in the Pearl River basin, a trivariate copula has been used to model the dependence between two consecutive monthly SPEI and the climate index. It has been found that consistent with the preliminary correlation analysis between each month climate index and SPEI series, the estimated transition probability matrix varied under different conditions of each climate index, and the regional responses of the transition probability matrix on each climate index are also different. These indicated that the trivariate copula is useful 
for the evaluation of the influences of climate indices on the transition probabilities of Markov Chain, and it is also very necessary as the field patterns of $850 \mathrm{hPa}$ wind anomalies and $500 \mathrm{hPa}$ vertical velocity anomalies with respect to each climate index have further verified the influences of climate indices on the precipitation.

Besides, to evaluate the influence of climate index on the drought characteristic, the mean extreme drought durations under different conditions of each climate index have been calculated in the study. Results indicated that for the Western part of the Pearl River basin, the mean extreme drought duration tends to be longer in the positive phase of ENSO while shorter in the positive phase of NAO and vice versa; for the Central part of the Pearl River basin, the mean extreme drought duration tends to be shorter in the positive phase of ENSO, NAO and PDO while longer in the positive phase of IOD and vice versa; for the Eastern part of the Pearl River basin, the mean extreme drought duration tends to be shorter in the positive phase of ENSO and PDO, and vice versa. This information will be of great importance for the mitigation of drought, and provide useful information for the agriculture planners and water resource management to plan possible measure on lessening the hazard of drought in the Pearl River basin, China.

However, the method to encompass the climate index into the models structure of Markov chain by the trivariate copula still need to be further analyzed. Based on a simple score measure, the enhancement in forecasting drought transition probabilities with respect to the case where the climate index is neglected has been quantified by Bonaccorso et al. (2015), however, there are no large improvement, 
and the result has also been found in the study (results not shown in the paper). These may be owing to the uncertainty caused by the limited sample size, and the uncertainty of copula evaluation related to the limited sample size has been analyzed by Serinaldi (2013) and Zhang et al. (2015), results shown that when the sample size is small, the goodness-of-fit tests cannot discriminate between alternative models because of the lack of power, especially for the trivariatie copula.

Acknowledgments: This work is financially supported by the National Science Foundation for Distinguished Young Scholars of China (Grant No.: 51425903), the Natural Foundation of Anhui Province (Grant No.: 1508085MD65), Project supported by the Funds for International Cooperation and Exchange of the National Natural Science Foundation of China (Grant No. 51210013), and is fully supported by a grant from the Research Grants Council of the Hong Kong Special Administrative Region, China (Project No. CUHK441313). Requirements of the data please write to Dr. Qiang Zhang: zhangq68@mail.sysu.edu.cn. Our cordial gratitude should be extended to the editor, Prof. Dr. Andras Bardossy, and anonymous reviewers for their professional and pertinent comments and suggestions which are greatly helpful for further improvement of the quality of this paper.

\section{References}

AghaKouchak, A., 2014. A baseline probabilistic drought forecasting framework using standardized soil moisture index: application to the 2012 United States drought. Hydrol. Earth Syst. Sci. 18(7), 2485-2492. 
Bonaccorso, B., A. Cancelliere, and G. Rossi, 2015. Probabilistic forecasting of drought class transitions in Sicily (Italy) using Standardized Precipitation Index and North Atlantic Oscillation Index. Journal of Hydrology 526, 136-150.

Cai, W., P. van Rensch, T. Cowan, and H. H. Hendon, 2011. Teleconnection Pathways of ENSO and the IOD and the Mechanisms for Impacts on Australian Rainfall Journal of Climate 24(15), 3910-3923.

Cancelliere, A., G. D. Mauro, B. Bonaccorso, and G. Rossi, 2007. Drought forecasting using the Standardized Precipitation Index. Water Resources Management 21(5), 801-819.

Chakak, A., and K. J. Koehler, 1995. A strategy for constructing multivariate distributions. Communications in Statistics-Simulation and Computation 24(3), $537-550$.

Chen, S. T., T. C. Yang, C. M. Kuo, C. H. Kuo, and P. S. Yu, 2013. Probabilistic drought forecasting in Southern Taiwan using El Niño-Southern Oscillation Index. Terr. Atmos. Ocean. Sci. 24(5), 911-924.

Chen, W., J. Feng, and R. G. Wu, 2013. Roles of ENSO and PDO in the Link of the East Asian Winter Monsoon to the following Summer Monsoon. Journal of Climate 26(2), 622-635.

Chen, Y. D., Q. Zhang, M. Z. Xiao, and V. P. Singh, 2015. Transition probability behaviors of drought events in the Pearl River basin, China, Stochastic Environmental Research and Risk Assessment, DOI 10.1007/ s00477-015-1178-2. 
Dai, A., 2013. The influence of the inter-decadal Pacific oscillation on US precipitation during 1923-2010. Climate Dynamics 41(3-4), 633-646.

Genest, C., I. Kojadinovic, J. Nešlehová, and J. Yan, 2011. A goodness-of-fit test for bivariate extreme-value copulas. Bernoulli 17(1), 253-275.

Kao, S. C., and R. S. Govindaraju, 2008. Trivariate statistical analysis of extreme rainfall events via the Plackett family of copulas. Water Resources Research 44, W02415.

Kao, S. C., and R. S. Govindaraju, 2010. A copula-based joint deficit index for droughts Journal of Hydrology 380(2), 121-134.

Linderholm, H. W., T. H. Ou, J. H. Jeong, C. K. Folland, D. Y. Gong, H. B. Liu, Y. Liu, and D. L. Chen, 2011. Interannual teleconnections between the summer North Atlantic Oscillation and the East Asian summer monsoon. Journal of Geophysical Research: Atmospheres 116(D13107).

Madadgar, S., and H. Moradkhani, 2013. A Bayesian Framework for Probabilistic Seasonal Drought Forecasting. Journal of Hydrometeorology 14(6), 1685-1705.

Madadgar, S., and H. Moradkhani, 2014. Spatio-temporal drought forecasting within Bayesian networks. Journal of Hydrology 512, 134-146.

Moore, G. W. K., I. A. Renfrew, and R. S. Pickart, 2013. Multidecadal Mobility of the North Atlantic Oscillation. Journal of Climate 26(8), 2453-2466.

Nelsen, R. B., 2006. An introduction to copulas, Springer Verlag, New York.

Paulo, A., and L. Pereira, 2007. Prediction of SPI Drought Class Transitions Using Markov Chains. Water Resources Management 21(10), 1813-1827. 
Peterson, T. C., M. P. Hoerling, P. A. Stott, and S. C. Herring, 2013. Explaining Extreme Events of 2012 from a Climate Perspective. Bulletin of the American Meteorological Society 94(9), S1-S74.

Renard, B., and M. Lang, 2007. Use of a Gaussian copula for multivariate extreme value analysis: Some case studies in hydrology. Advances in Water Resources, 30(4): 897-912.

Salvadori, G., 2007. Extremes in nature: an approach using copulas, Springer Verlag, New York.

Salvadori, G., and C. De Michele, 2006. Statistical characterization of temporal structure of storms. Advances in Water Resources 29(6), 827-842.

Sanusi, W., A. Jemain, W. Zin, and M. Zahari, 2015. The Drought Characteristics Using the First-Order Homogeneous Markov Chain of Monthly Rainfall Data in Peninsular Malaysia. Water Resources Management 29(5), 1523-1539.

Serinaldi, F., 2013. An uncertain journey around the tails of multivariate hydrological distributions. Water Resources Research 49(10), 6527-6547.

Vicente-Serrano, S. M., S. Beguería, and J. I. López-Moreno, 2010. A Multiscalar Drought Index Sensitive to Global Warming: The Standardized Precipitation Evapotranspiration Index. Journal of Climate 23(7), 1696-1718.

Wilhite, D. A., 2000. Drought as a natural hazard: concepts and definitions, in Drought: A Global Assessment., edited by D. A. Wilhite, pp. 3-18, Routledge Publishers, London.

Wilhite, D. A., M. V. K. Sivakumar, and R. Pulwarty, 2014. Managing drought risk 
in a changing climate: The role of national drought policy. Weather and Climate Extremes 3, 4-13.

Xiao, M. Z., Q. Zhang, and V. P. Singh, 2015. Influences of ENSO, NAO, IOD and PDO on seasonal precipitation regimes in the Yangtze River basin, China. International Journal of Climatology 35, 3556-3567.

Yuan, X., E. F. Wood, N. W. Chaney, J. Sheffield, J. Kam, M. Liang, and K. Guan, 2013. Probabilistic Seasonal Forecasting of African Drought by Dynamical Models. Journal of Hydrometeorology 14(6), 1706-1720.

Yuan, Y., H. Yang, W. Zhou, and C. Y. Li, 2008. Influences of the Indian Ocean dipole on the Asian summer monsoon in the following year. International Journal of Climatology 28(14), 1849-1859.

Zhang, Q., M. Z. Xiao, and V. P. Singh, 2015. Uncertainty evaluation of copula analysis of hydrological droughts in the East River basin, China. Global and Planetary Change 129, 1-9.

Zhang, Q., M.Z. Xiao, V.P. Singh, and J.F. Li, 2012. Regionalization and spatial changing properties of droughts across the Pearl River basin, China. Journal of Hydrology 472, 355-366.

Zhang, Q., M. Z. Xiao, V. P. Singh, and X. H. Chen, 2013. Copula-based risk evaluation of hydrological droughts in the East River basin, China. Stochastic Environmental Research and Risk Assessment 27(6), 1397-1406.

Zhang, Q., M.Z. Xiao, V.P. Singh, and Y.D. Chen, 2014. Max-stable based evaluation of impacts of climate indices on extreme precipitation processes across the 
Poyang Lake basin, China. Global and Planetary Change 122, 271-281. 


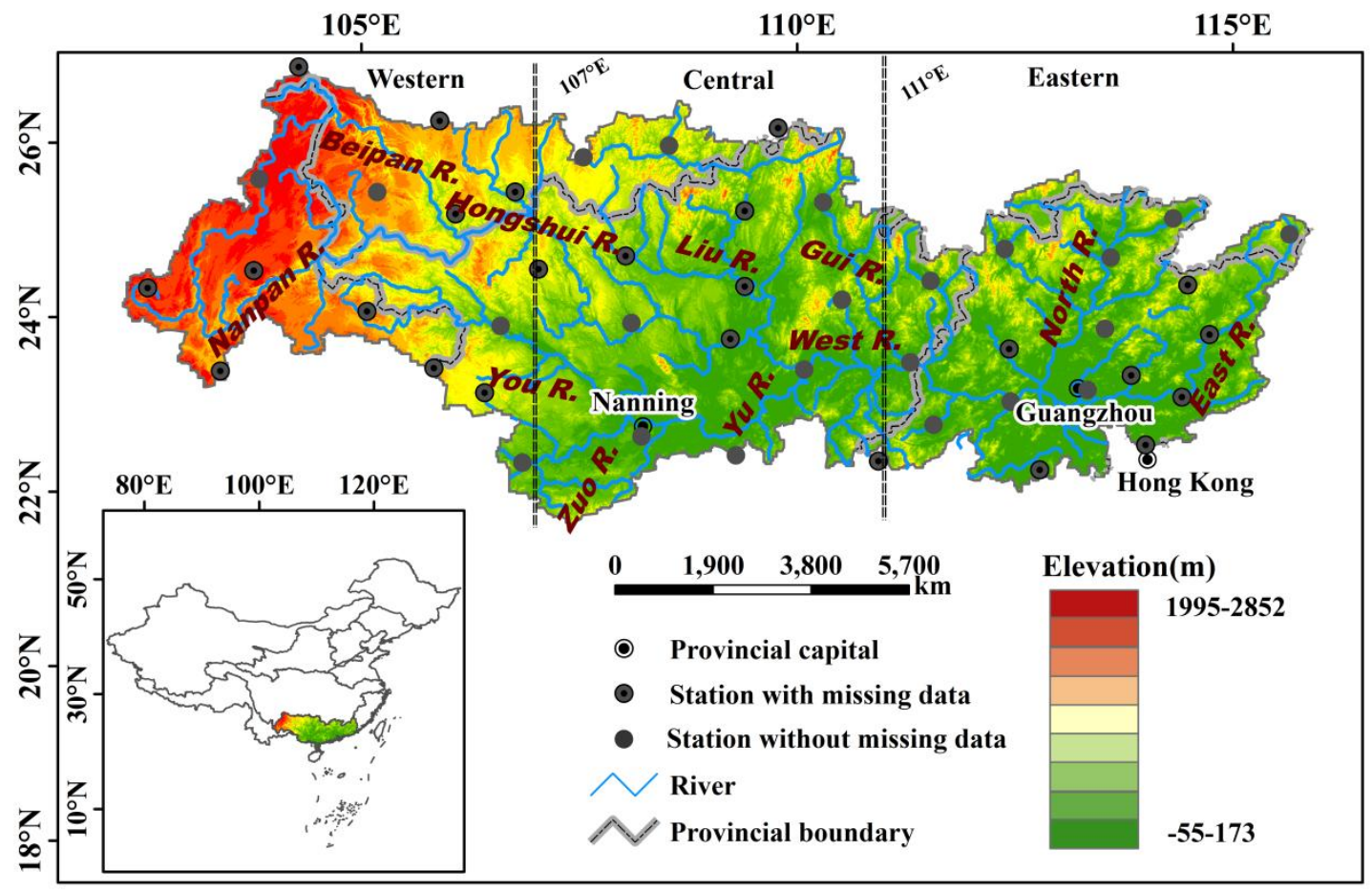

Fig. 1 Study region and locations of meteorological stations, and the region has been divided into three sub-regions that the Western, Central and Eastern part. 

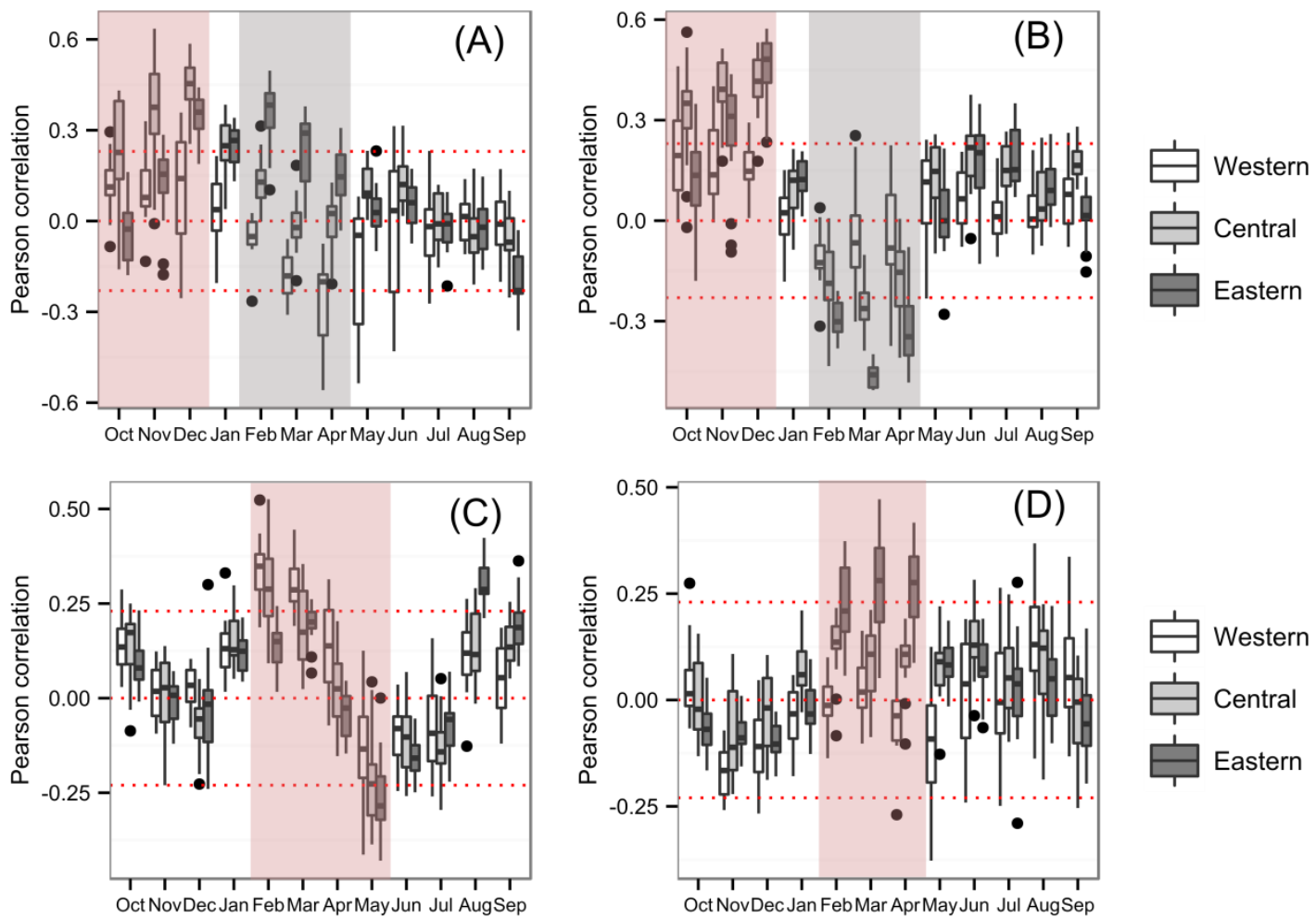

Fig. 2 Pearson correlation between each month climate index and the SPEI in the next month at different sub-regions in the Pearl River basin, where (A) for ENSO; (B) for IOD; (C) for NAO and (D) for PDO. The thresholds at the 95\% confidence interval are shown as red dotted lines. 

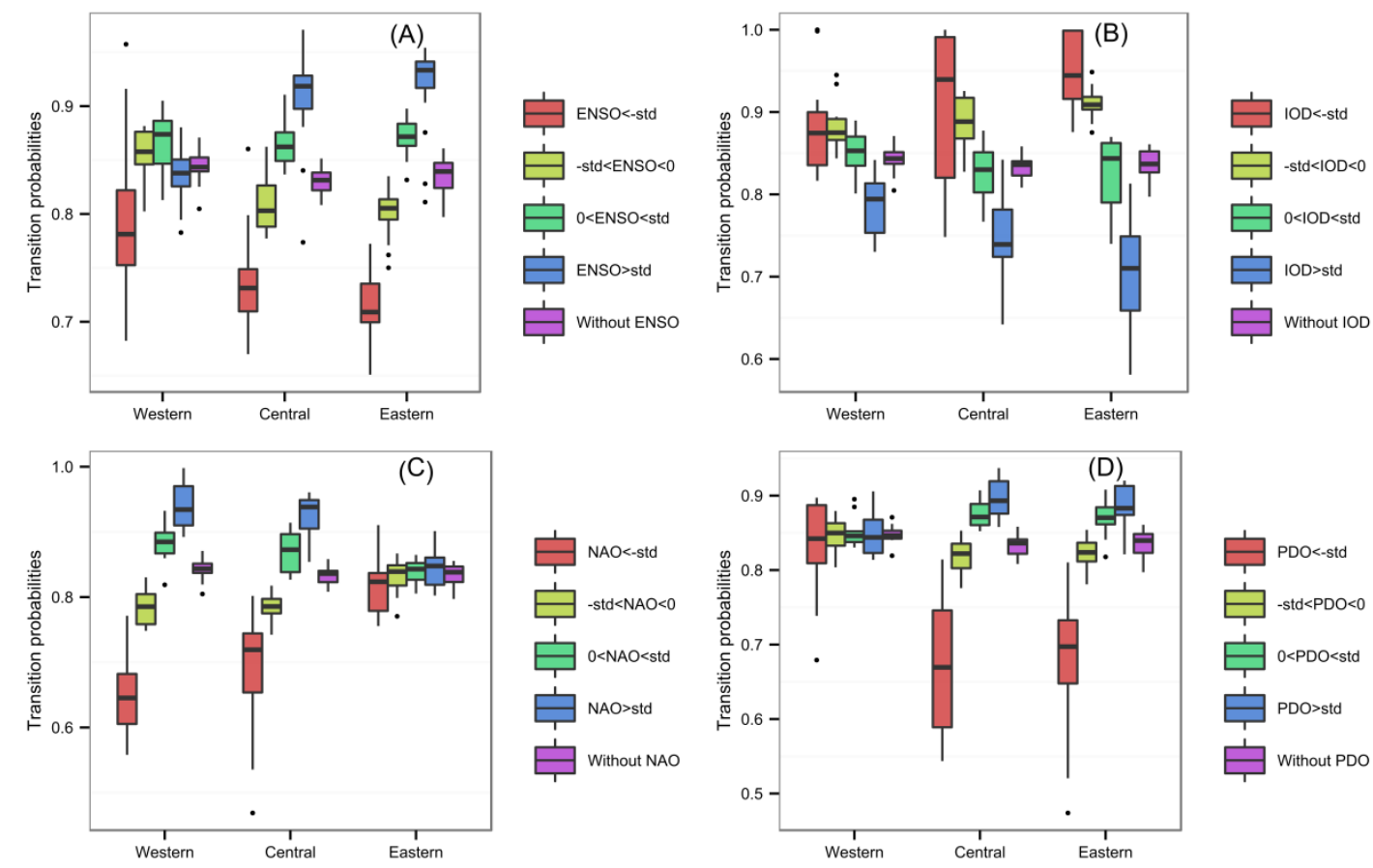

Fig. 3 Transition probabilities from No drought to No drought in March at different sub-regions under different conditions of each climate index, where (A) for ENSO; (B) for IOD; (C) for NAO and (D) for PDO. The "std" in the legend means the standard deviation of each climate index in March. 

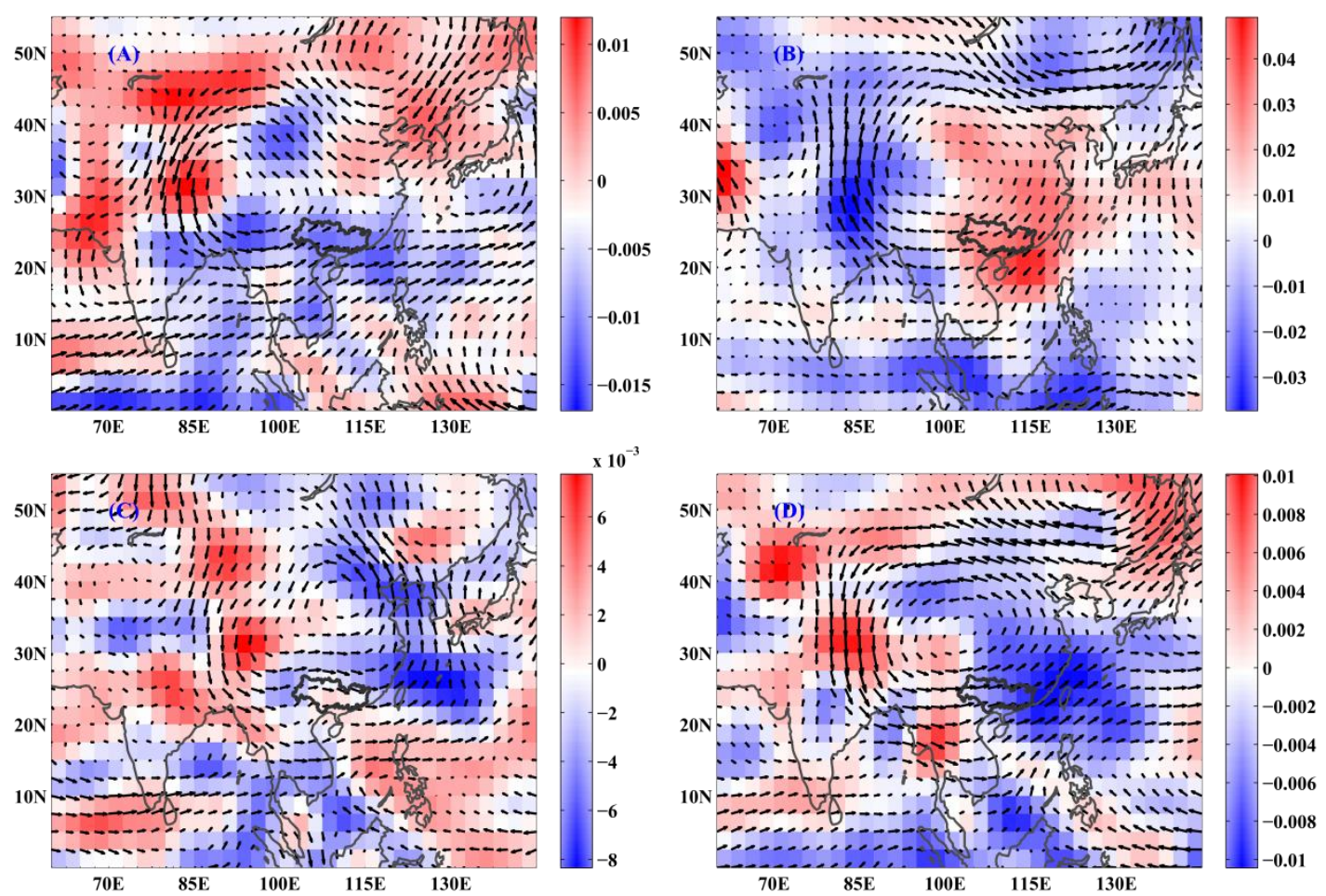

Fig. 4 Regression anomalous patterns of wind (vectors, unit: $\mathrm{m} / \mathrm{s}$ ) at $850 \mathrm{hPa}$ and vertical velocity at $500 \mathrm{hPa}$ (shading, unit: $\mathrm{P} / \mathrm{s}$ ) in April with respect to each climate index in March, where (A) for ENSO; (B) for IOD; (C) for NAO and (D) for PDO. 

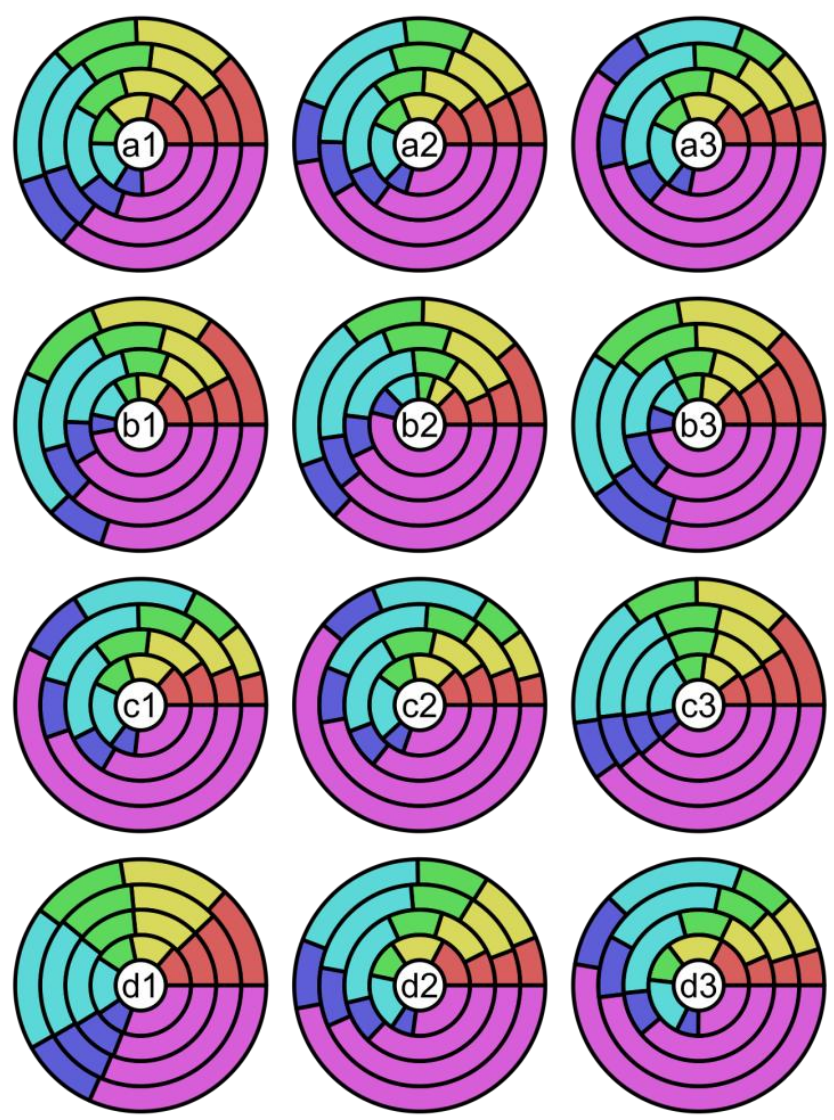

\section{- D4 - D3 - D2 - D1 - D0 - No drought}

Fig. 5 The forecasted regional average occurrence probabilities of drought in April under different conditions of each climate index, where (a) for ENSO; (b) for IOD; (c) for NAO and (d) for PDO, and for the suffix of the letter, (1) for the Western part; (2) for the Central part and (3) for the Eastern part. The rings from the inside to the outside represent the conditions of $(-\infty,-s t d],(-s t d, 0],(0$, std] and $(\operatorname{std},-\infty)$ for each climate index respectively, and the "std" means the standard deviation of each climate index in March. 

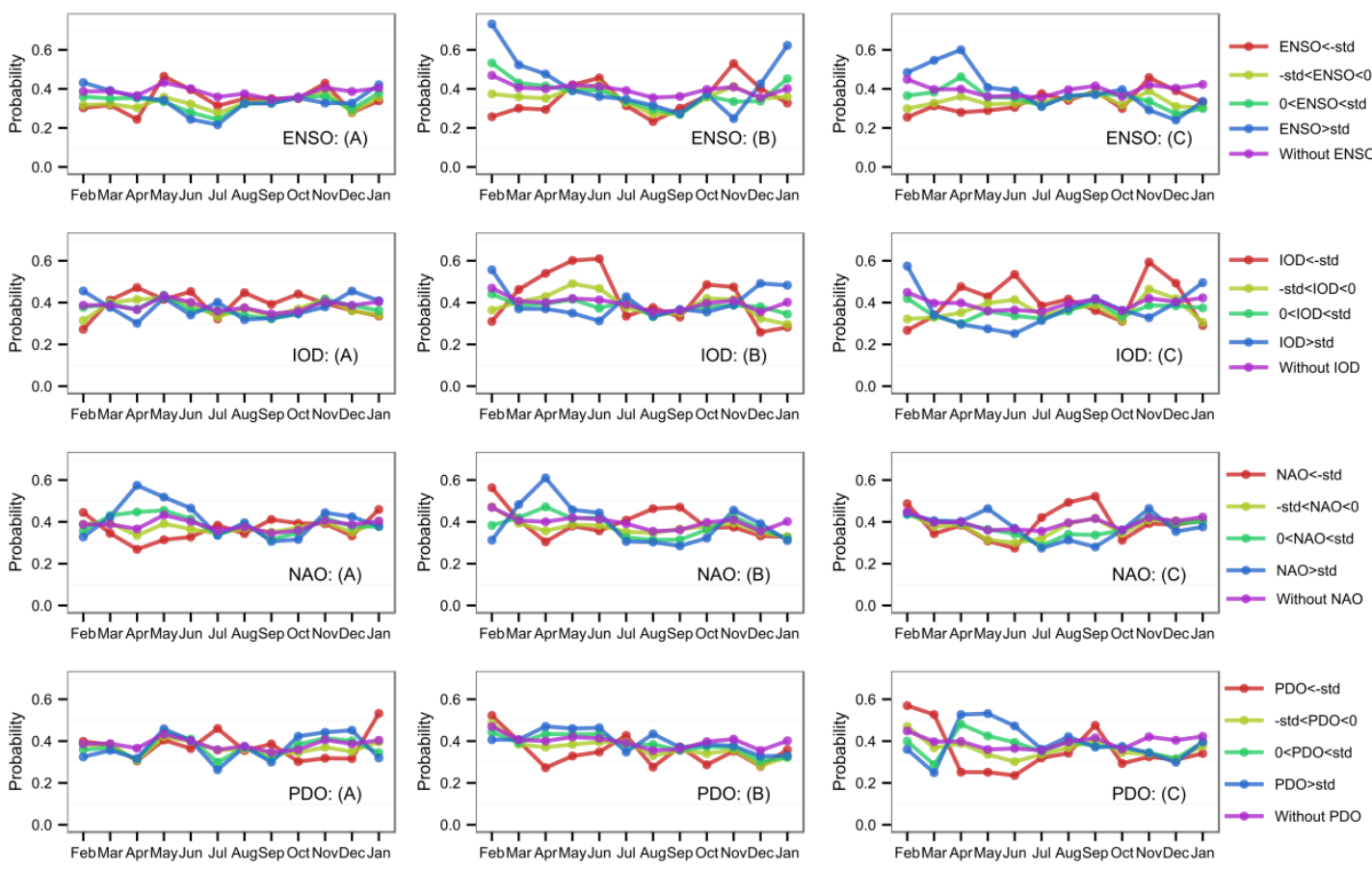

Fig. 6 The forecasted regional average occurrence probabilities of No drought in each month under different conditions of each climate index, where (A) for the Western part; (B) for the Central part and (C) for the Eastern part. The forecasted occurrence probabilities are estimated with the initial probability of each drought class is the same in the previous month. The "std" in the legend means the standard deviation of each climate index in each month. 

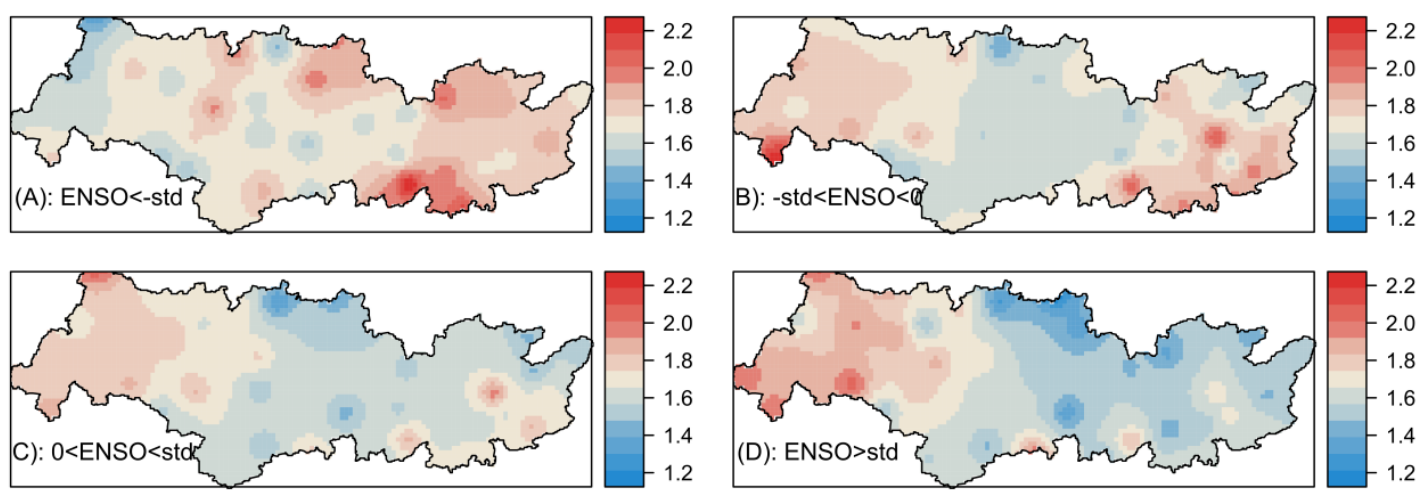

Fig. 7 Spatial distribution of the mean duration of extreme drought under different conditions of ENSO in the Pearl River basin. The unit of the color bar is month, and the "std" in the legend means the standard deviation of ENSO in each month. 

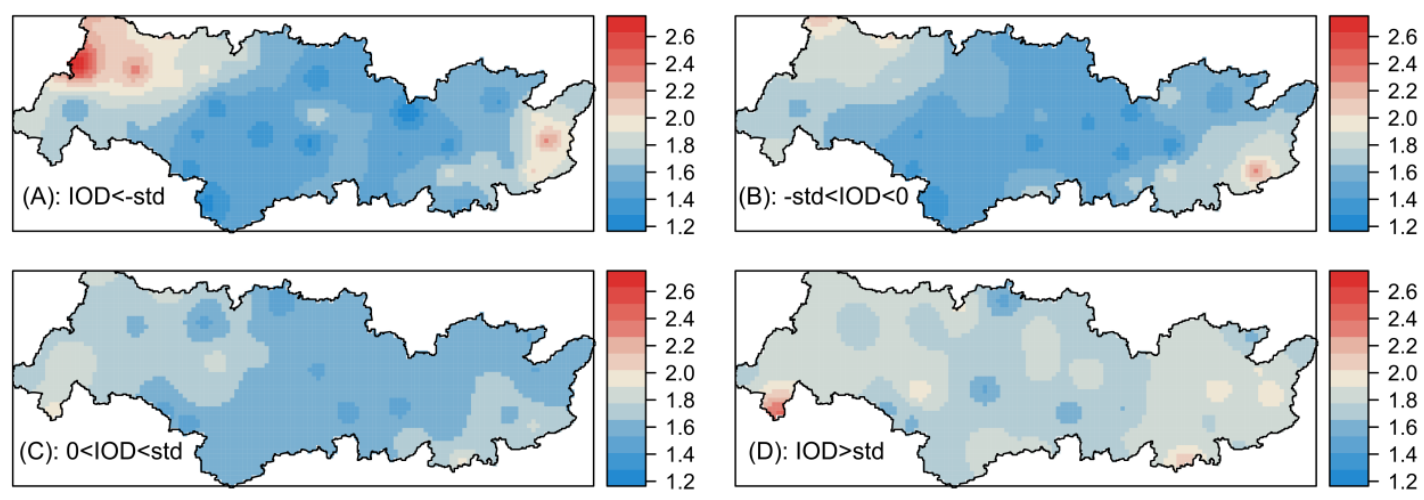

Fig. 8 Spatial distribution of the mean duration of extreme drought under different conditions of IOD in the Pearl River basin. The unit of the color bar is month, and the "std" in the legend means the standard deviation of IOD in each month. 

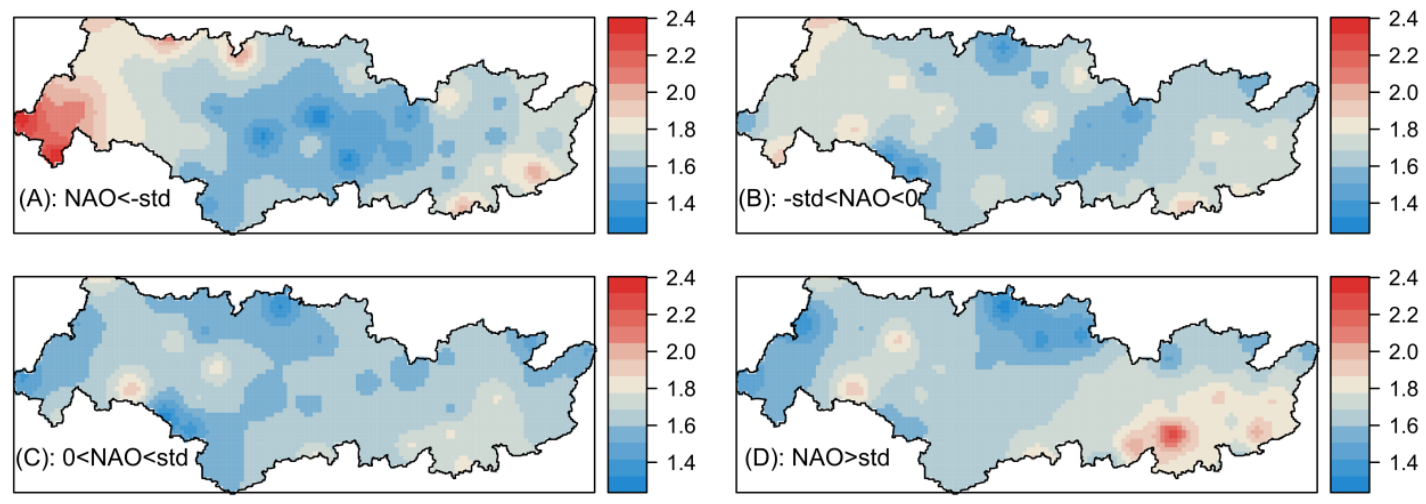

Fig. 9 Spatial distribution of the mean duration of extreme drought under different conditions of NAO in the Pearl River basin. The unit of the color bar is month, and the "std" in the legend means the standard deviation of NAO in each month. 

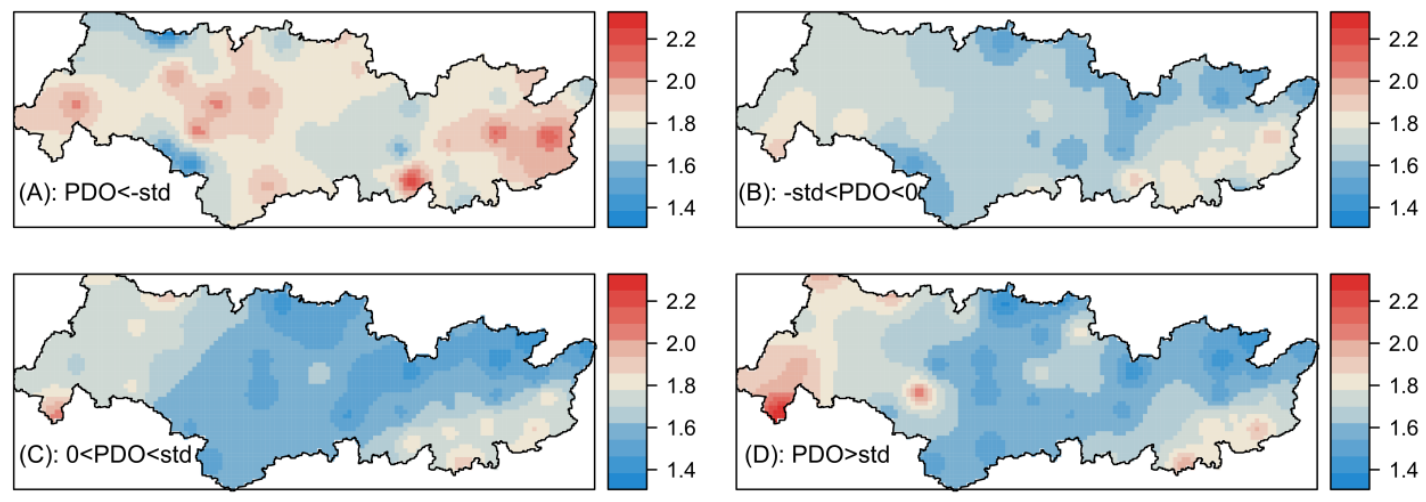

Fig. 10 Spatial distribution of the mean duration of extreme drought under different conditions of PDO in the Pearl River basin. The unit of the color bar is month, and the "std" in the legend means the standard deviation of PDO in each month. 
Table 1 Drought classifications based on SPEI, similar to the drought classification defined by the U.S. Drought Monitor.

\begin{tabular}{lll}
\hline Drought status & Drought severity & SPEI value \\
\hline No drought & No drought & $>-0.5$ \\
D0 & Abnormally & $(-0.7,-0.5]$ \\
D1 & Moderate & $(-1.2,-0.7]$ \\
D2 & Severe & $(-1.5,-1.2]$ \\
D3 & Extreme & $(-2.0,-1.5]$ \\
D4 & Exceptional & $(-\propto,-2.0]$ \\
\hline
\end{tabular}

